\title{
How hot is the molecular gas in the Galactic Center?
}

\author{
Katharina Immer ${ }^{1}$, Jens Kauffmann ${ }^{2}$, Thushara Pillai ${ }^{2}$, \\ Adam Ginsburg ${ }^{1}$ and Karl M. Menten ${ }^{2}$ \\ ${ }^{1}$ European Southern Observatory \\ Karl-Schwarzschild-Strasse 2, 85748 Garching bei München, Germany \\ email: kimmer@eso.org \\ ${ }^{2}$ Max-Planck-Institut für Radioastronomie \\ Auf dem Hügel 69, 53121 Bonn, Germany
}

\begin{abstract}
The molecular clouds in the Central Molecular Zone of our Galaxy (CMZ; inner $\sim 200 \mathrm{pc}$ ) show systematically higher gas than dust temperatures ( $>50 \mathrm{~K}$ vs $<30 \mathrm{~K})$ in recent $\mathrm{H}_{2} \mathrm{CO}$ line and dust continuum surveys. This discrepancy is puzzling since gas and dust temperatures should become equal over short times at the high densities observed in these clouds. In deep $\mathrm{H}_{2} \mathrm{CO}(3-2)$ and (4-3) observations of seven clouds in the CMZ, we detected not only large temperature differences between the clouds but also large gradients within the clouds. Comparing the temperatures and the main $\mathrm{H}_{2} \mathrm{CO}$ lines at 218 and $291 \mathrm{GHz}$, we found a positive correlation between those two parameters, indicating that turbulence plays an important role in the heating of the gas. As a follow-up, we mapped the temperature tracers $\mathrm{CH}_{3} \mathrm{CCH}$ and $\mathrm{CH}_{3} \mathrm{CN}$ in these seven clouds to derive multiple temperature estimates and test the accuracy of high gas temperatures.
\end{abstract}

Keywords. Galaxy: center, ISM: molecules, ISM: structure, ISM: clouds, Submillimeter: ISM

\section{Motivation}

In the Central Molecular Zone (CMZ, inner 200 pc of our Galaxy), we can study star formation under extreme conditions. Here, the velocity dispersion, gas temperatures, magnetic field strengths, pressure, etc. are much higher than in the Galactic disk (Morris \& Serabyn 1996). Recent CMZ surveys have shown that the gas temperatures (> $50 \mathrm{~K}$; Güsten et al. 1981; Hüttemeister et al. 1993; Ao et al. 2013; Mills \& Morris 2013; Ott et al. 2014; Ginsburg et al. 2016; Immer et al. 2016) in the Galactic center clouds are much higher than the dust temperatures ( $<20 \mathrm{~K}$; Lis et al. 1999; Molinari et al. 2011).

While many previous gas temperature measurements are based on observations of the ammonia molecule which traces low-density gas $\left(\mathrm{n} \sim 10^{3} \mathrm{~cm}^{-3}\right)$, we used the $\mathrm{H}_{2} \mathrm{CO}$ thermometer for our gas temperature study of $\mathrm{CMZ}$ clouds. $\mathrm{H}_{2} \mathrm{CO}$ is a slightly asymmetric rotor molecule. It has two different species (i.e. ortho and para) whose differences in the level populations are due to collisions. Comparing the relative intensities of para$\mathrm{H}_{2} \mathrm{CO}$ lines within the same $\mathrm{K}_{a}$ ladder gives estimates of the gas temperature (Mangum $\&$ Wootten 1993). Since the transitions of the $K_{a}$ ladders are close in frequency, they can be observed within one spectrum, making them calibration-independent. These gas temperature measurements and the further results are published in Immer et al. (2016)

To test if the $\mathrm{H}_{2} \mathrm{CO}$ temperatures are representative of the bulk of the CMZ gas or if there is a selection bias towards warmer temperatures or higher densities in our $\mathrm{H}_{2} \mathrm{CO}$ results, we observed the temperature tracers $\mathrm{CH}_{3} \mathrm{CN}$ and $\mathrm{CH}_{3} \mathrm{CCH}$ towards the same sample of clouds. Both molecules have transitions that are sensitive to very hot gas if there is any. The analysis of this data is ongoing (Immer et al., in prep.). 


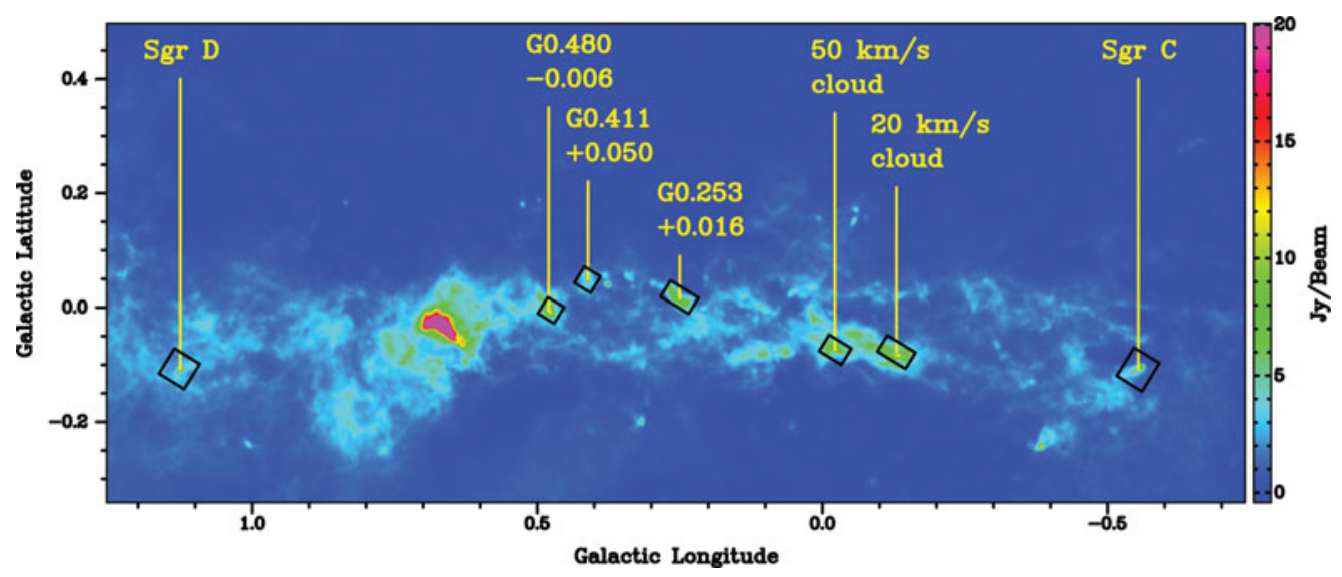

Figure 1. $870 \mu \mathrm{m}$ emission of the CMZ from the ATLASGAL survey (Schuller et al. 2009). The targets of our temperature study are marked. The boxes show the sizes of the observed OTF maps. Figure adopted from Immer et al. 2016a.

\section{Observations}

In 2012 and 2014, we mapped the $\mathrm{H}_{2} \mathrm{CO}(3-2)$ and (4-3) multiplets at 218 and 291 $\mathrm{GHz}$ with the Swedish Heterodyne Facility Instrument (SHeFI, Vassilev et al. 2008) and First Light APEX Submillimeter Heterodyne (FLASH) receiver (Heyminck et al. 2006; Klein et al. 2014) at the Atacama Pathfinder EXperiment (APEX†; Güsten et al. 2006) telescope towards five and seven CMZ clouds (Fig. 1), respectively. In 2015, we mapped the same seven clouds in the $\mathrm{CH}_{3} \mathrm{CN} / \mathrm{CH}_{3} \mathrm{CCH}$ ladders at $165 / 170 \mathrm{GHz}$ and $202 / 205$ GHz with Band 5 of the Swedish-ESO PI receiver at APEX (SEPIA; Immer et al. 2016b). The data are smoothed to a velocity resolution of $1 \mathrm{~km} \mathrm{~s}^{-1}$. The beam sizes at 170, 205, 218 , and $291 \mathrm{GHz}$ are $37^{\prime \prime}, 31^{\prime \prime}, 30^{\prime \prime}$, and $24^{\prime \prime}$, respectively.

\section{3. $\mathrm{H}_{2} \mathrm{CO}$ gas temperature measurements}

To measure the gas temperatures in our clouds, we produced integrated intensity maps of the transitions $\mathrm{H}_{2} \mathrm{CO}\left(3_{2,1}-2_{2,0}\right), \mathrm{H}_{2} \mathrm{CO}\left(3_{0,3}-2_{0,2}\right), \mathrm{H}_{2} \mathrm{CO}\left(4_{2,2}-3_{2,1}\right)$, and $\mathrm{H}_{2} \mathrm{CO}\left(4_{0,4}-\right.$ $3_{0,3}$ ) for all velocity components of our targets (Fig. 2 for the $8-14 \mathrm{~km} \mathrm{~s}^{-1}$ velocity component of the $20 \mathrm{~km} / \mathrm{s}$ cloud) . Then, for each pixel in these maps, we determined the

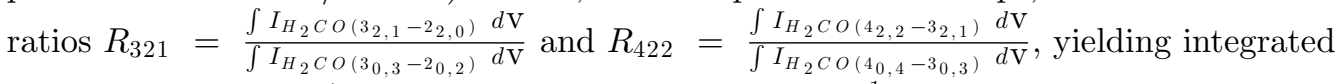
intensity ratio maps (see Fig. 3, left, for the $8-14 \mathrm{~km} \mathrm{~s}^{-1}$ velocity component of the 20 $\mathrm{km} / \mathrm{s}$ cloud). We used RADEX (van der Tak et al. 2007) to create model intensities for the para- $\mathrm{H}_{2} \mathrm{CO}$ lines from which we then calculated the same ratios $\mathrm{R}_{321}$ and $\mathrm{R}_{422}$. In the end, we yield functions which give estimates of the kinetic gas temperatures for given $\mathrm{R}_{321}$ and $\mathrm{R}_{422}$. Combining our integrated intensity ratio maps with the radiative transfer models, we yield two independent gas temperature maps for each velocity component of each target (see Fig. 3, right, for the $8-14 \mathrm{~km} \mathrm{~s}^{-1}$ velocity component of the $20 \mathrm{~km} / \mathrm{s}$ cloud; for more details of this analysis, please see Immer et al. 2016a).

Consistent with previous surveys, we measure high gas temperatures above $50 \mathrm{~K}$ in all our sources. In the temperature maps, we detect clear temperature gradients. This is an indication that heating mechanisms that act uniformly on the bulk of the molecular

$\dagger$ APEX is a collaboration between the Max-Planck-Institut für Radioastronomie, the European Southern Observatory, and the Onsala Space Observatory. 

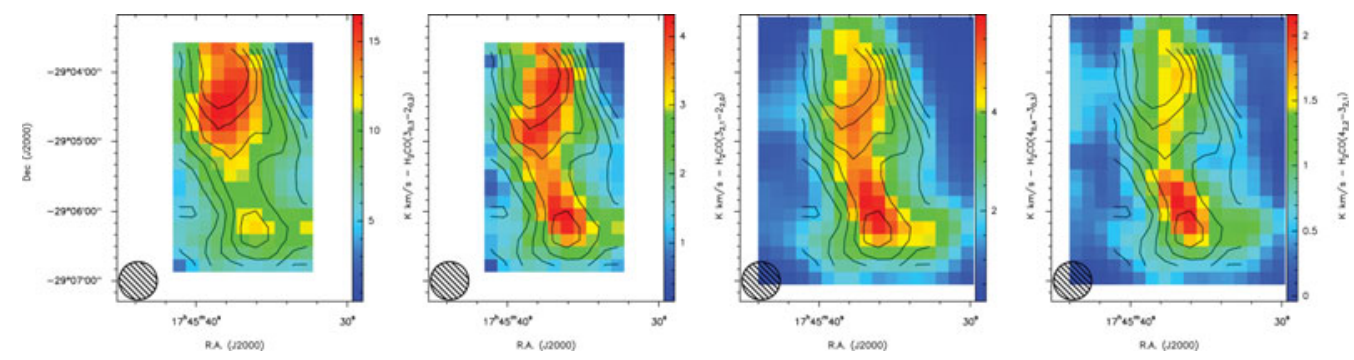

Figure 2. $\mathrm{H}_{2} \mathrm{CO}$ integrated intensity maps (from left to right: $\mathrm{H}_{2} \mathrm{CO}\left(3_{0,3}-2_{0,2}\right)$, $\mathrm{H}_{2} \mathrm{CO}\left(3_{2,1}-2_{2,0}\right)$, $\mathrm{H} 2 \mathrm{CO}\left(4_{0,4}-3_{0,3}\right)$, and $\left.\mathrm{H}_{2} \mathrm{CO}\left(4_{2,2}-3_{2,1}\right)\right)$ for the $8-14 \mathrm{~km} \mathrm{~s}^{-1}$ component of the $20 \mathrm{~km} / \mathrm{s}$ cloud. The contours show the moment 0 map of the $\mathrm{H}_{2} \mathrm{CO}\left(3_{0,3}-2_{0,2}\right)$ transition, produced over the whole velocity range of the source (levels: $30 \%-90 \%$ of the maximum in steps of $10 \%$ ). The circle in the lower left corner shows the $33^{\prime \prime}$ beam. Figure adopted from Immer et al. 2016a.
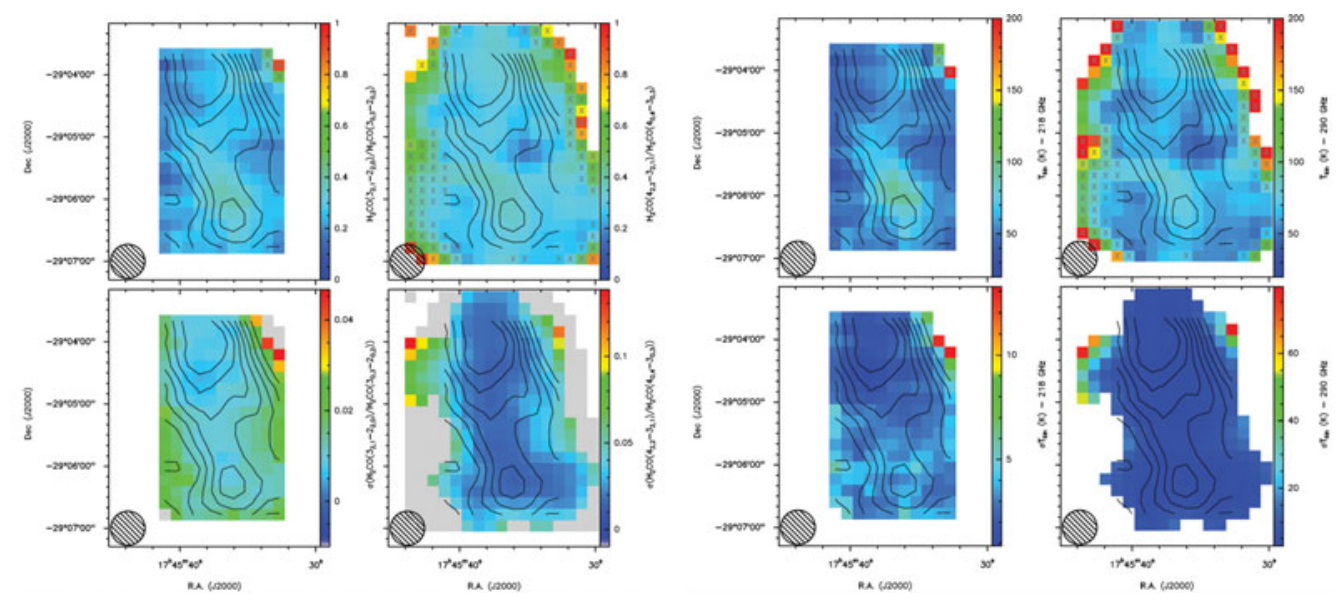

Figure 3. Left: $\mathrm{H}_{2} \mathrm{CO}$ ratio (upper panels) and uncertainty maps (lower panels) (left: $\mathrm{R}_{321}$, right: $\mathrm{R}_{422}$ ). Right: $\mathrm{H}_{2} \mathrm{CO}$ temperature (upper panels) and uncertainty maps (lower panels), derived from the $218 \mathrm{GHz}$ (left) and $291 \mathrm{GHz}$ data (right). Contours and beam as in Fig. 2. Figure adopted from Immer et al. 2016a.

gas cannot play an important role in the heating of the gas. We found a clear positive correlation between the line widths of the main $\mathrm{H}_{2} \mathrm{CO}$ lines at 218 and $291 \mathrm{GHz}$ and the measured temperatures which points towards turbulence being one of the main heating agents of the gas.

\section{4. $\mathrm{CH}_{3} \mathrm{CN} / \mathrm{CH}_{3} \mathrm{CCH}$ gas temperature measurements}

The $\mathrm{CH}_{3} \mathrm{CN}(9-8)$ and (11-10) ladders at 165 and $202 \mathrm{GHz}$, respectively, and the $\mathrm{CH}_{3} \mathrm{CCH}(10-9)$ and (12-11) ladders at 170 and $205 \mathrm{GHz}$, respectively, are detected towards all our sources. In some sources, we even detect the $\mathrm{CH}_{3} \mathrm{CN}(12-11)$ transitions at $220 \mathrm{GHz}$ which were observed in the same band as the $\mathrm{H}_{2} \mathrm{CO} 218 \mathrm{GHz}$ transitions. In Fig. 4, we show the $\mathrm{CH}_{3} \mathrm{CN}$ ladder at $165 \mathrm{GHz}$ and the rotational temperature diagram of the $36-42 \mathrm{~km} \mathrm{~s}^{-1}$ component of G0.253+0.016 towards the temperature peak of the source. Five, four and four $\mathrm{CH}_{3} \mathrm{CN}$ transitions are detected at 165,202 , and $221 \mathrm{GHz}$, respectively, and fitted. The rotational temperature diagram shows that a low and a high temperature component is necessary to fit the data. In the future, we will model the detected lines in each pixel of the maps with the XCLASS software (Möller et al. 2015) by 

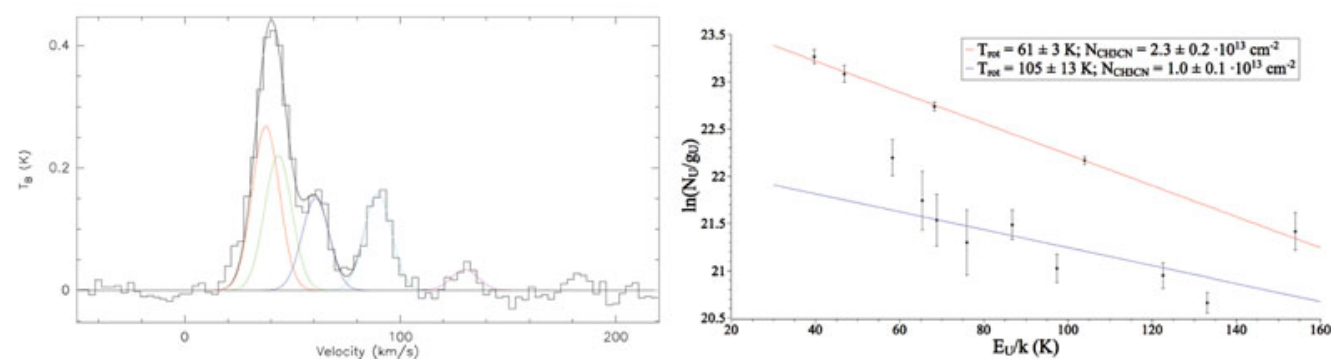

Figure 4. Left: Spectrum of $\mathrm{CH}_{3} \mathrm{CN}(9-8)$ at $165 \mathrm{GHz}$ towards the temperature peak of G0.253+0.016. Five transitions are detected and fitted. Right: Rotational temperature diagram towards the same position from all detected $\mathrm{CH}_{3} \mathrm{CN}$ transitions at 165,202 , and $220 \mathrm{GHz}$. A low and a high temperature component are necessary to fit the data.

solving the radiative transfer equation. Thus, we will produce independent temperature maps for $\mathrm{CH}_{3} \mathrm{CN}$ and $\mathrm{CH}_{3} \mathrm{CCH}$ for all velocity components of all our sources.

\section{References}

Ao, Y., Henkel, C., Menten, K. M., et al. 2013, A\&GA, 550, A135

Ginsburg, A., Henkel, C., Ao, Y., et al. 2016, A\&A, 586, A50

Güsten, R., Nyman, L. A., Schilke, P., et al. 2006, A\&SA, 454, L13

Güsten, R., Walmsley, C. M., \& Pauls, T. 1981, A\&A, 103, 197

Heyminck, S., Kasemann, C., Güsten, R., de Lange, G., \& Graf, U. U. 2006, A\&A, 454, L21

Hüttemeister, S., Wilson, T. L., Bania, T. M., \& Martin-Pintado, J. 1993, A\&SA, 280, 255

Immer, K., Kauffmann, J., Pillai, T., et al. 2016, ArXiv e-prints (1607.03535)

Immer, K., Belitsky, V., Olberg, M., et al. 2016, The Messenger, 165, 13

Klein, T., Ciechanowicz, M., Leinz, C., et al. 2014, IEEE Transactions on Terahertz Science and Technology, 4, 588

Lis, D. C., Li, Y., Dowell, C. D., \& Menten, K. M. 1999, in ESA Special Publication, Vol. 427, The Universe as Seen by ISO, ed. P. Cox \& M. Kessler, 627

Mangum, J. G. \& Wootten, A. 1993, ApJS, 89, 123

Mills, E. A. C. \& Morris, M. R. 2013, ApJ, 772, 105

Möller, T., Endres, C., \& Schilke, P., 2015, ArXiv e-prints (1508.04114)

Molinari, S., Bally, J., Noriega-Crespo, A., et al. 2011, ApJ, 735, L33

Morris, M. \& Serabyn, E. 1996, ARAA, 34, 645

Ott, J., Weiß, A., Staveley-Smith, L., Henkel, C., \& Meier, D. S. 2014, ApJ, 785, 55

Schuller, F., Menten, K. M., Contreras, Y., et al. 2009, A\&A A, 504, 415

van der Tak, F. F. S., Black, J. H., Schöier, F. L., Jansen, D. J., \& van Dishoeck, E. F. 2007, $A \mathscr{E} A, 468,627$

Vassilev, V., Meledin, D., Lapkin, I., et al. 2008, A\&A, 490, 1157 